1 Hacettepe Journal of Mathematics and Statistics

h Volume 45 (2) (2016), 373-379

\title{
Null controllability of heat equation with switching controls under Robin's boundary condition
}

\author{
Ali Hamidoğlu*
}

\begin{abstract}
In this paper, we consider the null controllability of 1-d heat equation endowed with Robin's boundary conditions, when the operator $-\frac{d^{2}}{d x^{2}}$ has positive eigenvalues and try to find sufficient conditions for building switching controls. In [1], the author developed a first analysis of this problem with Dirichlet's boundary conditions and obtain sufficient conditions for switching controls. We firstly consider 1-d heat system endowed with two controls. Then we try to build switching control strategies guaranteeing that, at each instant of time, only one control is activated.
\end{abstract}

Keywords: Heat equation, Robin's boundary condition, variational approach, switching control.

2000 AMS Classification: 93B05, 93C20.

Received: 11.12.2014 Accepted: 15.04.2015 Doi : 10.15672/HJMS.20164512501

\section{Introduction}

First of all, we define the general problem of controllability in PDEs. Meanly, it consists in investigating whether the solution of the PDE can be driven to a given final target by means of a control. More precisely, the controllability problem may be defined as follows. Consider an evolution system with given a time interval $t \in(0, T)$, initial and final states. We try to find a suitable control such that the solution matches both the initial state at time $t=0$ and the final one at time $t=T$. This is a type of exact controllability problem. There are other type of controllability problems beside that exact one. For instance, when the final target is achieved to zero, then the system is null controllable or when the set of reachable states (set of final targets) is dense in the space where the evolution system is satisfied, then the system is approximate controllable. These different concepts coincide in finite dimensional space. Because, in finite-dimensional space the only close affine dense subspace is the whole space itself. But this is no longer the case in the context of PDE. Indeed, in infinite- dimensional

*Department of Mathematics, Middle East Technical University, Ankara/Turkey. Email: ali.hamidoglu@metu.edu.tr. 
spaces we can easily find strict dense subspaces, while in finite-dimension they do not exist. These are classical problems in control theory and there is a large literature on the topic. We refer for instance to the book by Lee and Marcus [8] for an introduction in the context of finite-dimensional systems. We also refer to the survey article by Russell [5], the articles by Zuazua [3, 4] and to the SIAM Review article and the book of Lions $[6,7]$ for an introduction to the controllability of PDE, also referred to as Distributed Parameter Systems.

This paper deals with some of new results in null controllability of 1-d heat equation with switching controls under Robin's boundary condition. We firstly consider the 1-d heat equation endowed with two boundary controls and lumped controls under Robin's boundary condition, when the operator $-\frac{d^{2}}{d x^{2}}$ has positive eigenvalues respectively, and then we will obtain sufficient conditions for building switching controls. To do this we introduce a new functional based on the adjoint system whose minimizers yield the switching controls. We show that, due to the time analyticity of solutions, under suitable conditions on the location of the controllers, switching control strategies exist in the 1-d heat equation under Robin's boundary condition.

\section{Boundary Controls}

Consider the heat equation in the space interval $(0,1)$ with two controls located at the extremes $x=0,1$ and satisfying Robin's boundary condition (RBC)

$$
\begin{cases}y_{t}-y_{x x}=0, & 0<x<1,0<t<T \\ y_{x}(0, t)-a_{0} y(0, t)=u_{0}(t), & 0<t<T \\ y_{x}(1, t)+a_{1} y(1, t)=u_{1}(t), & 0<t<T \\ y(x, 0)=y^{0}(x), & 0<x<1\end{cases}
$$

We consider the problem of null controllability. More precisely, given an initial datum $y_{0} \in L^{2}(0,1)$ we look for controls $u_{0}(t), u_{1}(t) \in L^{2}(0, T)$ such that $y(x, T)=0$ and satisfying switching property

$$
u_{0}(t) u_{1}(t)=0, \quad \text { a.e. } t \in(0, T) .
$$

It is worth to mention the fact that whenever a system is controllable, the control can be constructed by minimizing a suitable quadratic functional defined on the class of solutions of the adjoint system (see e.g. [1], [3], [4]).

For $\varphi^{0}$ in $L^{2}(0,1)$, we consider the solution $\varphi:[0,1] \times[0, T] \rightarrow C\left([0, T], L^{2}(0,1)\right)$, of the following backward Cauchy linear problem ${ }^{\dagger}$

$$
\begin{cases}\varphi_{t}+\varphi_{x x}=0, & 0<x<1,0<t<T, \\ \varphi_{x}(0, t)-a_{0} \varphi(0, t)=0, & 0<t<T, \\ \varphi_{x}(1, t)+a_{1} \varphi(1, t)=0, & 0<t<T, \\ \varphi(x, T)=\varphi^{0}(x), & 0<x<1 .\end{cases}
$$

This linear system is called the adjoint system corresponding to the 1 -d heat equation with Robin's boundary condition. We know that (see e.g. [2]) the Fourier representation of solutions of the adjoint system with positive eigenvalues are of the form:

$$
\varphi(x, t)=\sum_{k \geq 1} \beta_{k} e^{\mu_{k}^{2}(t-T)} \omega_{k}(x)
$$

†The class of solutions $\varphi$ of backward Cauchy linear problem is class of smoother functions. 
where

$$
\omega_{k}(x)=\cos \mu_{k} x+\frac{a_{0}}{\mu_{k}} \sin \mu_{k} x .
$$

Now, for obtaining switching controls, we consider the following quadratic functional (see, e.g. [1]),

$$
J_{s}^{\alpha}\left(\varphi^{0}\right)=\frac{1}{2} \int_{0}^{T} \max \left\{|\varphi(0, t)|^{2},|\alpha \varphi(1, t)|^{2}\right\} d t+\int_{0}^{1} y^{0}(x) \varphi(x, 0) d x
$$

where $\alpha \in \mathbb{R}$ and minimize (2.6) over the class $\mathcal{H}$ of initial data given by

$$
\mathcal{H}=\left\{\varphi^{0}: \int_{0}^{T}\left[|\varphi(0, t)|^{2}+|\varphi(1, t)|^{2}\right] d t<\infty\right\}
$$

where $\varphi(x, t)$ is the solution of the adjoint system (2.3) associated to the final state $\varphi^{0}$. This space endowed with the canonical norm

$$
\left\|\varphi^{0}\right\|_{\mathcal{H}}=\left[\int_{0}^{T}|\varphi(0, t)|^{2}+|\varphi(1, t)|^{2} d t\right]^{\frac{1}{2}} .
$$

constitutes a Hilbert space. Let us analyse the positivity of the norm $\left\|\varphi^{0}\right\|_{\mathcal{H}}$ in space $\mathcal{H}$. Here we will use (2.4), (2.5) as a Fourier representation of the solution of (2.3). Therefore we have

$$
\int_{0}^{T}|\varphi(0, t)|^{2}+|\varphi(1, t)|^{2} d t \geq \int_{0}^{T}|\varphi(0, t)|^{2} d t=\int_{0}^{T}\left|\sum_{k \geq 1} \beta_{k} e^{\mu_{k}^{2}(t-T)}\right|^{2} d t
$$

Observe that

$$
\begin{aligned}
\|\varphi(x, 0)\|_{L^{2}(0,1)}^{2} & =\int_{0}^{1} \varphi^{2}(x, 0) d x=\int_{0}^{1}\left|\sum_{k \geq 1} \beta_{k} e^{-\mu_{k}^{2} T} \omega_{k}(x)\right|^{2} d x \\
& \leq C \sum_{k \geq 1} \beta_{k}^{2} e^{-2 k^{2} T}
\end{aligned}
$$

where $C>0$, which is independent from $\left\{\beta_{k}\right\}_{k \geq 1}$.

Now, we will give very important lemma on families of real exponentials. This lemma is known as estimates on families of real exponentials (see, e.g. [1],[4]).

2.1. Lemma. In our case, it is guaranteed that

$$
\int_{0}^{T}\left|\sum_{k \geq 1} \beta_{k} e^{k^{2}(t-T)}\right|^{2} d t \geq c_{1} \sum_{k \geq 1} e^{-2 k^{2} T} \beta_{k}^{2}
$$

for suitable positive constants $c_{1}>0$ which is independent from $\left\{\beta_{k}\right\}_{k \geq 1}$.

By using Lemma 2.1 in (2.7) and comparing with inequality (2.8), we will have the following observability inequality:

$$
\|\varphi(x, 0)\|_{L^{2}(0,1)}^{2} \leq \hat{C} \int_{0}^{T}|\varphi(0, t)|^{2}+|\varphi(1, t)|^{2} d t
$$

for positive constant $\hat{C}>0$ which is independent from $\left\{\beta_{k}\right\}_{k \geq 1}$.

The functional $J_{s}^{\alpha}: \mathcal{H} \longrightarrow \mathbb{R}$ is well defined, continuous, and strictly convex. ${ }^{\ddagger}$ For checking the coercivity property, one should prove that

$$
\lim _{\left\|\varphi^{0}\right\|_{L^{2}(0,1)} \rightarrow \infty} \frac{J_{s}^{\alpha}\left(\varphi^{0}\right)}{\left\|\varphi^{0}\right\|_{L^{2}(0,1)}} \geq \epsilon
$$

${ }^{\ddagger}$ This comes from the observability inequality (2.9) 
For this to be true, the unique continuation property of the adjoint system (2.3) suffices (see e.g. [1]). Namely,

$$
\mu\{t \in(0, T):|\varphi(0, t)|=|\varphi(1, t)|\}>0 \Rightarrow \varphi \equiv 0 .
$$

2.2. Lemma. Assume that

$$
|\alpha| \neq\left[\frac{\mu_{k}^{2}+a_{1}^{2}}{\mu_{k}^{2}+a_{0}^{2}}\right]^{\frac{1}{2}}, \quad \forall k \geq 1,
$$

holds. Then, (2.10) satisfy for solution of the adjoint system (2.3).

Proof. Firstly, assume that $\mu\{t \in(0, T):|\varphi(0, t)|=|\varphi(1, t)|\}>0$. We show that under the assumption of (2.11), we have $\varphi \equiv 0$. We know that (see e.g. [2]) positive eigenvalues $\left\{\mu_{k}\right\}_{k \geq 1}$ of adjoint system (2.3) satisfy eigenvalue equation:

$$
\tan \left(\mu_{k}\right)=\frac{\left(a_{0}+a_{1}\right) \mu_{k}}{\mu_{k}^{2}-a_{0} a_{1}}
$$

and also we would have

$$
(k-1) \pi<\mu_{k}<k \pi \quad \text { and } \quad \lim _{k \rightarrow \infty} \mu_{k}-(k-1) \pi=0, \quad(k=1,2,3,4, \ldots) .
$$

Now assume that $\mu(I)>0$, using again the Fourier representation of solution (2.4) of (2.3), we have

$$
\varphi(0, t) \pm \alpha \varphi(1, t)=\sum_{k \geq 1} \beta_{k} e^{\mu_{k}^{2}(t-T)}\left(1 \pm \alpha\left(\cos \mu_{k}+\frac{a_{0}}{\mu_{k}} \sin \mu_{k}\right)\right)
$$

The function $\varphi(0, t) \pm \alpha \varphi(1, t)$ is time analytic for $t \leq T$. Consequently, if they vanish for a set of time instants of positive measure, then they vanish for all $t \leq T$. It is then easy to see, by multiplying above identity by the real exponentials $e^{-\eta^{2}(t-\bar{T})}$ successively, starting from $\eta=1$ and taking limits as $t \rightarrow-\infty$ that

$$
\beta_{k}\left(1 \pm \alpha\left(\cos \mu_{k}+\frac{a_{0}}{\mu_{k}} \sin \mu_{k}\right)\right)=0, \quad \forall k \geq 1 .
$$

To conclude that $\beta_{k}=0$ for all $k \geq 1$, it is sufficient to have that

$$
1 \pm \alpha\left(\cos \mu_{k}+\frac{a_{0}}{\mu_{k}} \sin \mu_{k}\right) \neq 0
$$

Assume the converse of (2.13), then we have

$$
\begin{aligned}
\alpha\left(\cos \mu_{k}+\frac{a_{0}}{\mu_{k}} \sin \mu_{k}\right) \pm 1=0 \quad & \Longleftrightarrow\left[\alpha\left(\cos \mu_{k}+\frac{a_{0}}{\mu_{k}} \sin \mu_{k}\right)\right]^{2}=1 \\
& \Longleftrightarrow \alpha^{2}\left(1+2 \frac{a_{0}}{\mu_{k}} \tan \mu_{k}+\frac{a_{0}^{2}}{\mu_{k}^{2}} \tan ^{2} \mu_{k}\right)=1+\tan ^{2} \mu_{k}
\end{aligned}
$$

Now using eigenvalue equation (2.12) and after some simplification, finally we obtain the following

$$
\alpha^{2}\left(\mu_{k}^{2}+a_{0}^{2}\right)=\left(\mu_{k}^{2}+a_{1}^{2}\right) .
$$

Therefore to obtain the unique continuation property, it is suffice to assume that

$$
|\alpha| \neq\left[\frac{\mu_{k}^{2}+a_{1}^{2}}{\mu_{k}^{2}+a_{0}^{2}}\right]^{\frac{1}{2}}, \quad \forall k \geq 1 .
$$


Therefore, by using Lemma 2.2, we have that $J_{s}^{\alpha}$ admits an unique minimizer $\hat{\varphi}^{0} \in \mathcal{H}$. As a result, by using variational approach, we find our switching controls:

$$
u_{0}(t)=-\hat{\varphi}(0, t) 1_{S_{0}}, \quad u_{1}(t)=\alpha^{2} \hat{\varphi}(1, t) 1_{S_{1}} \quad \text { for } t \in(0, T)
$$

where

$$
\begin{gathered}
S_{0}=\{t \in(0, T):|\varphi(0, t)|>|\alpha \varphi(1, t)|\} \\
S_{1}=\{t \in(0, T):|\alpha \varphi(1, t)|>|\varphi(0, t)|\} .
\end{gathered}
$$

At the end, we obtain the following new result

2.3. Theorem. Given 1-d heat equation (2.1) under Robin's boundary condition, when the operator $-\frac{d^{2}}{d x^{2}}$ has positive eigenvalues, to obtain null controls that satisfying switching property (2.2), it is sufficient to assume that $\alpha$ satisfies the following relation

$$
|\alpha| \neq\left[\frac{\mu_{k}^{2}+a_{1}^{2}}{\mu_{k}^{2}+a_{0}^{2}}\right]^{\frac{1}{2}}, \quad \forall k \geq 1
$$

\section{Lumped Controls}

Let $f_{0}(x)$ and $f_{1}(x)$ be control profiles in $L^{2}(0,1)$. Consider the following heat equation

$$
\begin{cases}y_{t}-y_{x x}=u_{0}(t) f_{0}(x)+u_{1}(t) f_{1}(x), & 0<x<1,0<t<T, \\ y_{x}(0, t)-a_{0} y(0, t)=0, & 0<t<T \\ y_{x}(1, t)+a_{1} y(1, t)=0, & 0<t<T \\ y(x, 0)=y^{0}(x), & 0<x<1 .\end{cases}
$$

Here, we would consider the same problem, i.e., given an initial datum $y_{0} \in L^{2}(0,1)$ we are looking for controls $u_{0}(t), u_{1}(t) \in L^{2}(0, T)$ such that null controllability of heat equation holds, i.e, $y(x, T)=0$ and switching condition satisfies:

$$
u_{0}(t) u_{1}(t)=0, \quad \text { a.e. } \quad t \in(0, T)
$$

The null control of 1 -d heat equation may be computed by minimizing the quadratic functional (see e.g. [1]),

$$
\begin{aligned}
\hat{J}_{s}\left(\varphi^{0}\right)= & \frac{1}{2} \int_{0}^{T} \max \left[\left|\int_{0}^{1} f_{0}(x) \varphi(x, t) d x\right|^{2},\left|\int_{0}^{1} f_{1}(x) \varphi(x, t) d x\right|^{2}\right] d t \\
& -\int_{0}^{T} y^{0}(x) \varphi(x, 0) d x
\end{aligned}
$$

over the class $\tilde{\mathcal{H}}$ of initial data given by

$$
\tilde{\mathcal{H}}=\left\{\varphi^{0}: \int_{0}^{T}\left[\left|\int_{0}^{1} f_{0}(x) \varphi(x, t) d x\right|^{2}+\left|\int_{0}^{1} f_{1}(x) \varphi(x, t) d x\right|^{2}\right] d t<\infty\right\}
$$

which endowed with the canonical norm

$$
\left\|\varphi^{0}\right\|_{\tilde{\mathcal{H}}}^{2}=\int_{0}^{T}\left[\left|\int_{0}^{1} f_{0}(x) \varphi(x, t) d x\right|^{2}+\left|\int_{0}^{1} f_{1}(x) \varphi(x, t) d x\right|^{2}\right] d t
$$

At first, we will show that $\|\cdot\|_{\tilde{\mathcal{H}}}$ actually defines norm on $\tilde{\mathcal{H}}$. For this, it is enough to show the positivity of $\|\cdot\|_{\tilde{\mathcal{H}}}$. Observe that

$$
\left\|\varphi^{0}\right\|_{\tilde{\mathcal{H}}}^{2}=\int_{0}^{T}\left[\left|\sum_{k \geq 1} \beta_{k} e^{\mu_{k}^{2}(t-T)} f_{0, k}\right|^{2}+\left|\sum_{k \geq 1} \beta_{k} e^{\mu_{k}^{2}(t T)} f_{1, k}\right|^{2}\right] d t
$$


where

$$
f_{0}(x)=\sum_{k \geq 1} f_{0, k} \omega_{k}(x), \quad f_{1}(x)=\sum_{k \geq 1} f_{1, k} \omega_{k}(x),
$$

and using Lemma 2.1, we then get the following weighted observability inequality

$$
\left\|\varphi^{0}\right\|_{\tilde{\mathcal{H}}}^{2} \geq c_{1} \sum_{k \geq 1} e^{-2 \mu_{k}^{2} T}\left[\left|f_{0, k}\right|^{2}+\left|f_{1, k}\right|^{2}\right] \beta_{k}^{2}
$$

where positive constant $c_{1}$ is independent from $\left\{\beta_{k}\right\}_{k \geq 1}$.

In addition, since (3.1) is well posed, the functional $\hat{J}\left(\varphi^{0}\right)$ is obviously continuous in $\tilde{\mathcal{H}}$, the convexity (strictly) of $\hat{J}\left(\varphi^{0}\right)$ comes from the weighted observability inequality (3.3). As we know that (see e.g. [3]) null controllability in time $T$ implies (finite) approximate controllability in time $T$. This comes form the fact that all the range of the semi-group generated by the heat equation is reachable. Therefore, we first prove the approximate controllability of the heat system in time $T$ under some conditions. For this, we will construct the new functional very similar with previous one $\hat{J}_{s}$ and with the same coercivity property, allows building approximate controllers: for any $\epsilon>0$ and any $y^{1} \in L^{2}(0,1)$

$$
\begin{aligned}
\hat{J}_{\epsilon}\left(\varphi^{0}\right)= & \frac{1}{2} \int_{0}^{T} \max \left[\left|\int_{0}^{1} f_{0}(x) \varphi d x\right|^{2},\left|\int_{0}^{1} f_{1}(x) \varphi d x\right|^{2}\right] d t \\
& +\epsilon\left\|\left(I-\pi_{E}\right) \varphi^{0}\right\|_{L^{2}(0,1)}+\int_{0}^{1} \varphi^{0} y^{1} d x-\int_{0}^{1} y^{0}(x) \varphi(x, 0) d x
\end{aligned}
$$

where $E$ is finite dimensional subspace of $L^{2}(0,1)$ and $\pi_{E}$ denotes the ortogonal projection from $L^{2}(0,1)$ over $E$.

Our aim is to build approximate lumped controls that satisfy switching property (3.2). In other words, given $\epsilon>0$, we try to find (finite) approximate controls $u_{0}^{\epsilon}$ and $u_{1}^{\epsilon}$ such that the solution $y_{\epsilon}$ of heat equation satisfies the condition

$$
\left\|y_{\epsilon}(x, T)\right\|_{L^{2}(0,1)} \leq \epsilon
$$

3.1. Lemma. Assume that the following unique continuation property

$$
\mu\left\{t \in(0, T):\left|\int_{0}^{1} f_{0}(x) \varphi(x, t) d x\right|=\left|\int_{0}^{1} f_{1}(x) \varphi(x, t) d x\right|\right\}>0 \Rightarrow \varphi \equiv 0 .
$$

holds. Then the heat system (3.1) is approximate controllable.

For the proof of Lemma 3.1, one should first prove that the functional $\hat{J}_{\epsilon}$ is coercive in $\tilde{\mathcal{H}}$ which directly comes from the assumption (3.4) and at the end, by using variational approach, one could easily get approximate controls for (3.1) (see e.g. [3]). Therefore, from Lemma 3.1, to get approximate controls, we should prove (3.4). Using (2.4), we have

$$
\int_{0}^{1} f_{0}(x) \varphi(x, t) d x \pm \int_{0}^{1} f_{1}(x) \varphi(x, t) d x=\sum_{k \geq 1} \beta_{k} e^{\mu_{k}^{2}(t-T)}\left(f_{1, k} \pm f_{0, k}\right) .
$$

The function $\int_{0}^{1} \varphi(x, t)\left(f_{0}(x) \pm f_{1}(x)\right) d x$ is time analytic for $t \leq T$. Consequently, if they vanish for a set of time instants of positive measure, then they vanish for all $t \leq T$. It is then easy to see, by multiplying above identity by the real exponentials $e^{-\eta^{2}(t-T)}$ successively, starting from $\eta=1$ and taking limits as $t \rightarrow-\infty$, that

$$
\beta_{k}\left(f_{1, k} \pm f_{0, k}\right)=0, \quad \forall k \geq 1 \text {. }
$$

To conclude that $\beta_{k}=0$ for all $k \geq 1$, it is sufficient to assume that

$$
f_{1, k} \pm f_{0, k} \neq 0 \quad \forall k \geq 1 .
$$


Therefore under the condition (3.5), our functional $\hat{J}_{\epsilon}$ admits an unique minimizer $\hat{\varphi}^{0} \in$ $\tilde{\mathcal{H}}$. For every $\epsilon>0$, by using variational approach, we will obtain approximate switching controls. We would like to say that for each $\epsilon>0$, we must have the fact that $u_{0}^{\epsilon}(t)$ and $u_{1}^{\epsilon}(t)$ are uniformly bounded in $L^{2}(0, T)$. But under the condition on Fourier coefficients of the initial datum $y^{0}$

$$
\sum_{k \geq 1} \frac{e^{2 \mu_{k}^{2} T}}{\left|f_{1, k}\right|^{2}+\left|f_{2, k}\right|^{2}}\left|y_{k}^{0}\right|^{2}<\infty
$$

being satisfied, by using weighted observability inequality (3.3) one could easily prove that $u_{0}^{\epsilon}(t)$ and $u_{1}^{\epsilon}(t)$ are uniformly bounded in $L^{2}(0, T)$. Hence at the end, by using variational approach we obtain the following switching controls

$$
\begin{array}{lll}
u_{0}(t)=-\int_{0}^{1} f_{0}(x) \hat{\varphi}(x, t) d x, & u_{1}(t)=0, & \text { in } S_{0} \\
u_{1}(t)=-\int_{0}^{1} f_{1}(x) \hat{\varphi}(x, t) d x, & u_{0}(t)=0, & \text { in } S_{1}
\end{array}
$$

where

$$
\begin{gathered}
S_{0}=\left\{t \in(0, T):\left|\int_{0}^{1} f_{0}(x) \hat{\varphi}(x, t) d x\right|>\left|\int_{0}^{1} f_{1}(x) \hat{\varphi}(x, t) d x\right|\right\} \\
S_{1}=\left\{t \in(0, T):\left|\int_{0}^{1} f_{1}(x) \hat{\varphi}(x, t) d x\right|>\left|\int_{0}^{1} f_{0}(x) \hat{\varphi}(x, t) d x\right|\right\} .
\end{gathered}
$$

In conclusion, we obtain the following new result

3.2. Theorem. Assume that $f_{0}(x)$ and $f_{1}(x)$ are two control profiles in $L^{2}(0,1)$ and their Fourier coefficients satisfying (3.5). Let Fourier coefficients of the initial datum $y^{0}$ satisfy (3.6). Then, for all $T>0$, there exist switching controls (3.7) and (3.8) satisfying our switching condition (3.2) and solution of (3.1) satisfies

$$
y(x, T)=0
$$

i.e, null controllability is satisfied.

Acknowledgements. The author wishes to thank anonymous referee for inspiring comments.

\section{References}

[1] E. Zuazua, Switching control, J. Eur. Math. Soc. 13, pp. 85-117, 2011.

[2] Walter A. Strauss, Partial Differential Equations: An Introduction, Wiley, 1992.

[3] E. Zuazua, Controllability and observability of partial differential equations: Some results and open problems, In: Handbook of Differential Equations: Evolutionary Equations, Vol.3, pp. 527-621, 2006.

[4] E. Zuazua, S. Micu, On the controllability of a fractional order parabolic equation, SIAM J. Control Optim., Vol.44, pp. 1950-1972, 2006.

[5] D.L. Russell, Controllability and stabilizability theory for linear partial differential equations. Resent progress and open questions, SIAM Rev., Vol.20, pp. 639-739., 1978.

[6] J.L. Lions, Optimal Control of Systems Governed by Partial Differential Equations, SpringerVerlang Berlin Heidelberg, 1971.

[7] J.L. Lions, Exact Controllability, Stabilizability and Perturbations for Distributed Systems", SIAM Rev., Vol. 30, pp. 1-68, 1988.

[8] E.B. Lee, L. Markus, Foundations of Optimal Control Theory, John Wiley and Sons, Inc., New York, 1967. 
